\title{
Angewandte
}

Supporting Information

(c) Wiley-VCH 2013

69451 Weinheim, Germany

\section{A Directional Entropic Force Approach to Assemble Anisotropic Nanoparticles into Superlattices**}

Kaylie L. Young, Michelle L. Personick, Michael Engel, Pablo F. Damasceno, Stacey N. Barnaby, Reiner Bleher, Tao Li, Sharon C. Glotzer,* Byeongdu Lee,* and Chad A. Mirkin*

anie_201306009_sm_miscellaneous_information.pdf 
Supporting Information

\section{Materials and Methods}

1a. Synthesis

Chemicals and Materials. Gold (III) chloride trihydrate $\left(\mathrm{HAuCl}_{4} \cdot 3 \mathrm{H}_{2} \mathrm{O}, 99.9+\%\right)$, silver nitrate $\left(\mathrm{AgNO}_{3}\right.$, 99.9999\%), sodium borohydride $\left(\mathrm{NaBH}_{4}, 99.99 \%\right)$, sodium chloride $(\mathrm{NaCl}, 99.999 \%)$, sodium bromide $(\mathrm{NaBr}, 99.0+\%)$, L-ascorbic acid (AA, 99+\%), cetyltrimethylammonium chloride (CTAC, 25 wt.\% in $\mathrm{H}_{2} \mathrm{O}$ ), and cetyltrimethylammonium bromide (CTAB, 99\%) were purchased from Aldrich and used without further purification. Hydrochloric acid $(\mathrm{HCl}, 1 \mathrm{~mol} / \mathrm{L}$ volumetric solution) was purchased from Fluka and used without further purification.

Synthesis of Small Seed Particles. $7 \mathrm{~nm}$ diameter Au seeds were prepared by quickly injecting $0.60 \mathrm{~mL}$ of ice-cold, freshly prepared $\mathrm{NaBH}_{4}(10 \mathrm{mM})$ into a rapidly stirring solution containing $0.25 \mathrm{~mL}$ of $\mathrm{HAuCl}_{4}$ $(10 \mathrm{mM})$ and either $10.0 \mathrm{~mL}$ of CTAC $(100 \mathrm{mM})$ or $10.0 \mathrm{~mL}$ of CTAB $(100 \mathrm{mM})$. The seed solution was stirred for 1 minute and then left undisturbed for 2 hours.

Synthesis of Tetrahexahedra. Tetrahexahedra were synthesized via a previously published method. ${ }^{[1]} \mathrm{A}$ growth solution was prepared by consecutively adding $0.2 \mathrm{~mL}$ of $\mathrm{HCl}(1 \mathrm{M}), 0.5 \mathrm{~mL}$ of $\mathrm{HAuCl}_{4}(10 \mathrm{mM})$, $100 \mu \mathrm{L}$ of $\mathrm{AgNO}_{3}(10 \mathrm{mM})$, and $100 \mu \mathrm{L}$ of AA $(100 \mathrm{mM})$ to $10.0 \mathrm{~mL}$ of $100 \mathrm{mM} \mathrm{CTAB}$, swirling the solution after each addition. The $7 \mathrm{~nm}$ diameter CTAB-capped seeds (above) were serially diluted in 100 $\mathrm{mM}$ CTAB to generate a solution which was $1 / 100$ the concentration of the as-synthesized seed solution. Particle growth was initiated by adding $100 \mu \mathrm{L}$ of the diluted small seeds to the growth solution, which was then swirled and incubated at $30^{\circ} \mathrm{C}$ for 3 hours.

Synthesis of Rhombic Dodecahedra. Rhombic dodecahedra were synthesized via a slight modification of a previously published method. ${ }^{[2-3]} \mathrm{A}$ growth solution was prepared by consecutively adding $0.5 \mathrm{~mL} \mathrm{NaCl}$ (1 M), $0.21 \mathrm{~mL}$ of $\mathrm{HCl}(1 \mathrm{M}), 0.6 \mathrm{~mL}$ of $\mathrm{HAuCl}_{4}(10 \mathrm{mM}), 10 \mu \mathrm{L}$ of $\mathrm{NaBr}(10 \mathrm{mM}), 10 \mu \mathrm{L}$ of $\mathrm{AgNO}_{3}(10$ $\mathrm{mM})$, and $100 \mu \mathrm{L}$ of AA $(100 \mathrm{mM})$ to $10.0 \mathrm{~mL}$ of $40 \mathrm{mM} \mathrm{CTAC}$, swirling the solution after each addition. The $7 \mathrm{~nm}$ diameter CTAC-capped seeds (above) were serially diluted in $100 \mathrm{mM}$ CTAC to generate a solution which was $1 / 200$ the concentration of the as-synthesized seed solution. Particle growth was initiated by adding $100 \mu \mathrm{L}$ of the diluted small seeds to the growth solution, which was then swirled and left undisturbed on the bench top until the reaction was complete. The rhombic dodecahedra were purified by filtration using a syringe-driven filter with pore size $\leq 0.2 \mu \mathrm{m}$ (PALL Life Sciences Acrodisk LC syringe filter, $0.2 \mu \mathrm{m}$ pore PVDF membrane). ${ }^{[2]}$

Synthesis of Large Seed Particles. Larger seeds $(\sim 40 \mathrm{~nm}$ diameter) were prepared by consecutively adding $0.2 \mathrm{~mL} \mathrm{HAuCl}_{4}(10 \mathrm{mM})$ and $40.0 \mu \mathrm{LAA}(100 \mathrm{mM})$ to a solution containing $8.0 \mathrm{~mL} \mathrm{H}_{2} \mathrm{O}$ and 1.6 mL 100 mM CTAC. ${ }^{[3]}$ The CTAC-capped $7 \mathrm{~nm}$ diameter seed particles synthesized above were diluted in $100 \mathrm{mM}$ CTAC to generate a solution which was $1 / 10$ the concentration of the original seed solution. Growth of the larger seed particles was initiated by adding $100 \mu \mathrm{L}$ of the diluted $7 \mathrm{~nm}$ diameter seeds. The reaction was swirled immediately after the addition of the seeds and then left undisturbed on the bench top until the reaction was complete. 
Synthesis of Octahedra. Octahedra were synthesized via a previously published method. ${ }^{[3]}$ A growth solution was prepared by consecutively adding $150 \mu \mathrm{L}$ of $\mathrm{HAuCl}_{4}(10 \mathrm{mM})$ and $60-80 \mu \mathrm{L}$ of AA (100 $\mathrm{mM}$ ) to $10.0 \mathrm{~mL}$ of $10 \mathrm{mM} \mathrm{CTAB}$, swirling the solution after each addition. Particle growth was initiated by adding $0.5 \mathrm{~mL}$ of the large seeds to the growth solution, which was then swirled and incubated at 30 ${ }^{\circ} \mathrm{C}$ for 3 hours.

Synthesis of Truncated Cubes. Truncated cubes were synthesized via a previously published method. ${ }^{[3]} \mathrm{A}$ growth solution was prepared by consecutively adding $150 \mu \mathrm{L}$ of $\mathrm{HAuCl}_{4}(10 \mathrm{mM})$ and $110 \mu \mathrm{L}$ of AA $(100 \mathrm{mM})$ to $10.0 \mathrm{~mL}$ of $10 \mathrm{mM} \mathrm{CTAB}$, swirling the solution after each addition. Particle growth was initiated by adding $0.5 \mathrm{~mL}$ of the large seeds to the growth solution, which was then swirled and incubated at $30^{\circ} \mathrm{C}$ for 3 hours.

The conditions used for assembly of the anisotropic nanoparticles are summarized in Table S2.

\section{1b. Small Angle X-ray Scattering (SAXS)}

The solutions containing the nanoparticle superlattices were transferred to $1.5 \mathrm{~mm}$ quartz capillaries (Charles Supper Co.) for in situ characterization with SAXS. SAXS experiments were performed at undulator beamline 12ID-C (12 keV) and the Dupont-Northwestern-Dow Collaborative Access Team (DND-CAT) beamline of the Advanced Photon Source at Argonne National Laboratory in Lemont, IL. The sample-to-detector distance was such as to provide a detecting range for scattering vector $q=4 \pi(\sin \theta) / \lambda$ of $0.008<q<0.20 \AA^{-1}$. The scattering vector was calibrated using a silver behenate standard. The exposure times varied from $0.1-0.5 \mathrm{~s}$. Scattered radiation was detected with a CCD area detector and was radially averaged to produce 1-D plots of scattered intensity $\mathrm{I}(q)$ versus $q$. In the main text, the data are presented as plots of structure factor $\mathrm{S}(q)$ versus $q$ where $\mathrm{S}(q)$ was calculated as $\mathrm{I}(q) /\left|\mathrm{F}(q)^{2}\right|$ where $\mathrm{I}(q)$ is the background corrected scattering and $\left|\mathrm{F}(q)^{2}\right|$ is the form factor due to the scattering of the NPs in the unaggregated state. ${ }^{[4]}$

Calculation of Interparticle Distances. The distance between nearest neighbors (center-to-center) within the nanoparticle superlattices were calculated using the SAXS data as

$$
d_{N P}=\frac{1}{10} \frac{C}{q_{0}}
$$

where $d_{N P}$ is the distance in nanometers between two neighboring nanoparticles, $\mathrm{q}_{0}$ is the position of the first scattering peak in $1 / \AA$, and $C$ is a constant that correlates the distance between nearest neighbor NPs and the distance between the [hkl] places associated with the first allowed reflection. Values of $C$ for each unit cell symmetry discussed in this work have been reported previously ${ }^{[5]}$ and are summarized in the Table S1.

Table S1. Revelant variables used to calculate interparticle distances and lattice parameters for the colloidal crystals from the SAXS data. 


\begin{tabular}{|c|c|c|c|c|}
\hline Crystal Type & Space Group & $\begin{array}{c}\mathbf{1}^{\text {st }} \text { reflection } \\
\text { [hkl] }\end{array}$ & $\begin{array}{c}\text { Nearest } \\
\text { Neighbor }\end{array}$ & $\boldsymbol{C}$ \\
\hline FCC & 225 & {$[111]$} & {$[1 / 2,1 / 2,0]$} & $\sqrt{ }(6)^{*} \pi$ \\
\hline SC & 221 & {$[100]$} & {$[1,0,0]$} & $2 \pi$ \\
\hline BCC & 229 & {$[110]$} & {$[1 / 2,1 / 2,1 / 2]$} & $\sqrt{ }(6)^{*} \pi$ \\
\hline hexagonal & 191 & {$[100]$} & {$[1,0,0]$} & $4 \pi / \sqrt{ }(3)$ \\
\hline
\end{tabular}

Calculation of Crystal Domain Size. From the full-width half max (FWHM) of the qo peak in the SAXS patterns, the average crystalline domain size was calculated using the Debye-Scherrer equation ${ }^{[6]}$

$$
t=\frac{0.9 \lambda}{B \cos \theta}
$$

where $t$ is the diameter of the crystal in $\AA, \lambda$ is the wavelength of the scattered X-rays (1.24 $\AA$ ), and $B$ is the FWHM of the $\mathrm{q}_{0}$ peak in radians. The average domain size for each crystal reported in this work is summarized in Table S2.

Table S2. Summary of the assembly conditions, the interparticle spacing, and the average crystalline domain size of the nanoparticle superlattices that were synthesized.

\begin{tabular}{|l|l|l|l|l|l|l|}
\hline NP Shape & $\begin{array}{c}\text { NP Edge } \\
\text { Length } \\
\mathbf{( n m )}\end{array}$ & $\begin{array}{l}\text { Surfactant } \\
\text { Concentration } \\
\mathbf{( M )}\end{array}$ & $\begin{array}{l}\text { Crystallographic } \\
\text { Symmetry }\end{array}$ & $\left.\mathbf{q}_{\mathbf{0}} \mathbf{(}^{-\mathbf{1}}\right)$ & $\begin{array}{l}\mathbf{d}_{\mathrm{NP}} \\
\mathbf{( n m )}\end{array}$ & $\begin{array}{c}\text { Average } \\
\text { crystal domain } \\
\text { size } \mathbf{( n m})\end{array}$ \\
\hline RD & 36.3 & 0.08 M CTAC & FCC & 0.01031 & 74.6 & 966 \\
\hline T. Cubes & 46.6 & 0.1 M CTAB & SC & 0.01026 & 61.2 & 1501 \\
\hline Octahedra & 70.0 & 0.1 M CTAB & BCC & 0.00969 & 78.0 & 1014 \\
\hline THH & 41.2 & 0.1 M CTAB & hexagonal & 0.01014 & 71.5 & 999 \\
\hline
\end{tabular}

1c. Modeling of SAXS data

Reciprocal lattice. The scattering peak positions were confirmed using the PowerCell software package available free of charge from the Federal Institute for Materials Research and Testing (http://www.ccp14.ac.uk/ccp/web-mirrors/powdcell/a_v/v_1/powder/e_cell.html). PowderCell assumes a single point scatterer in each particle center. It does not consider the geometry of the polyhedral particles, nor lattice fluctuations or imperfections.

Powder diffraction. The structure factor $S(q)$ was numerically simulated in approximation by the formula $^{[7]}$

$$
S(q) \propto \sum_{h, k, l} \frac{1}{q_{h, k, l}{ }^{2}} \frac{\left|F\left(\boldsymbol{q}_{h, k, l}\right)\right|^{2}}{P\left(q_{h, k, l}\right)}\left|\sum_{n=1}^{N} \exp \left(i \boldsymbol{q}_{h, k, l} \boldsymbol{x}_{\boldsymbol{n}}\right)\right|^{2} \frac{1}{\left(q-q_{h, k, l}\right)^{2}+q_{\mathrm{L}}^{2}} \exp \left(\frac{q^{2}}{2 q_{\mathrm{DW}}{ }^{2}}\right),
$$

where we sum over the $N$ particles in the unit cell, and $\boldsymbol{q}_{h, k, l}=\boldsymbol{b}_{1} h+\boldsymbol{b}_{2} k+\boldsymbol{b}_{3} l$ is a reciprocal lattice vector using the reciprocal lattice basis $\left\{\boldsymbol{b}_{i}\right\}$. The form factors and its orientational averaged square are given by 


$$
F(\boldsymbol{q})=\int_{V} \exp \left(i \boldsymbol{q}_{h, k, l} \boldsymbol{x}\right) \mathrm{d}^{3} \boldsymbol{x} \quad \text { and } \quad P(q) \propto \oint|F(q \sin \theta \cos \varphi, q \sin \theta \sin \varphi, q \cos \theta)|^{2} \mathrm{~d} \Omega .
$$

We perform the integration over the polyhedral particle volume $V$ both analytically and using Monte Carlo integration. In Monte Carlo integration, scatterers are placed randomly and uniformly within the particles. Particle geometries and cell dimensions follow the data given in Table 1. Peak line shape is approximated by a Lorentzian. Thermal motion of the nanoparticles at their lattice sites and other structural fluctuations of the crystal lattice are estimated to be on the order of the available space to the next nearest neighbors and included as a Debye-Waller factor. Size polydispersity is also included in the calculation.

Comparison of calculated structure factors and SAXS data. The positions of the scattering peaks were analyzed by comparison with the PowderCell results. Experimental scattering curves are reproduced with good accuracy using numerical simulation. Both peak positions and relative peak intensities of experimental and numerical results agree well.

\section{1d. Electron Microscopy}

Scanning electron microscopy (SEM) images were obtained using a Hitachi SU8030 SEM. For cryoelectron microscopy studies, samples were drop-cast onto a $\mathrm{Cu}$ TEM grid and plunge-frozen using a Vitrobot MarkIII (FEI Inc.). The frozen samples were kept in liquid nitrogen, mounted in a cryo sample holder (Gatan Inc., Model 626), and analyzed at $-170{ }^{\circ} \mathrm{C}$ using a Hitachi HD-2300 STEM in z-contrast mode at an acceleration voltage of $80 \mathrm{kV}$.

\section{1e. Assembly Simulations}

Monte Carlo simulations were performed for hard rhombic dodecahedra, truncated cubes, (truncated) octahedra, and tetrahexahedra using the geometric shape and truncation amounts similar to those measured in experiments. Cubes were truncated by $25 \%$ (=amount of edge truncated), similar to the value found in experiment, and octahedra were truncated by $17 \%$, higher than that found in experiment (5\%). We believe this truncation captures the effective shape of the octahedra responsible for the depletion interaction better, because the covering of the polyhedral surface with surfactant ligands introduces an additional roundedness of the sharp octahedral tips when particle interact. For THH particles, we used an aspect ratio of 2.5, higher than that found in experiment $(\sim 2.0)$. In contrast, cubic THH with aspect ratio 1.0 assembled into FCC. We never observed elongated THH with aspect ratio of 1.5 and 2.0 to selfassemble by entropy alone and believe that stronger entropic forces (implemented via depletion interactions) are necessary to align rods of shorter aspect ratio. Nevertheless, the critical aspect ratio of rods for spontaneous parallel alignment is already shorter than the critical aspect ratio of spherocylinders, which suggests that the planar facets of THH enhance the entropic alignment.

Particles were initialized with random position and orientation in solution and observed to assemble by entropy maximization into FCC, simple cubic, BCC, and hexagonal lamellae, respectively. The first three structures agree with experimental observation, while the fourth is different from that surmised from SAXS data. The simulation results support the contention that the first three reported structures are entropically stabilized. For the THH system, we expect additional forces, including electrostatics and possibly van der Waals interactions, to play a role due to the more extreme anisotropy of this shape compared to the others studied. Crystal structures were identified by calculation of diffraction patterns, bond order diagrams, radial distribution function, structure factor, and by visualization. 


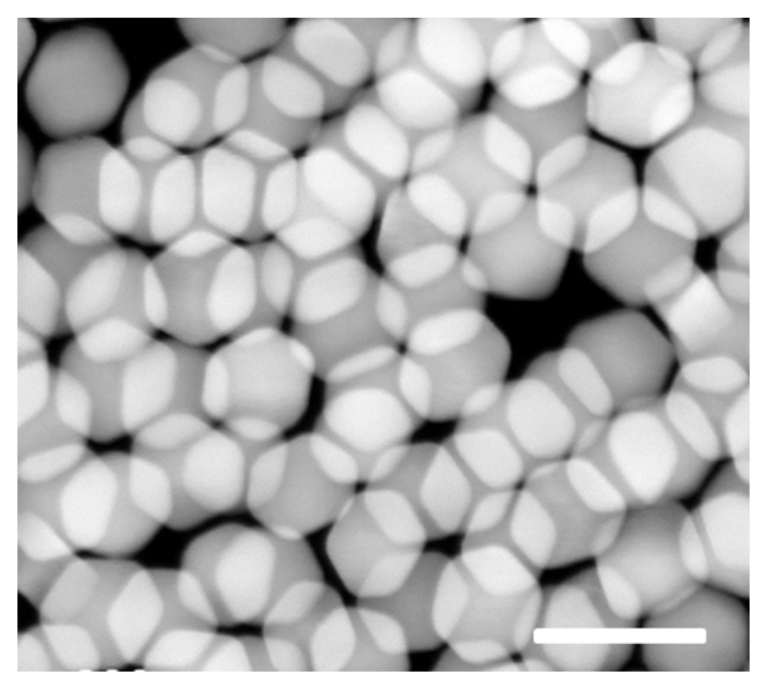

Figure S1. Cryo-electron microscopy image of rhombic dodecahedra (RD) assembled into an FCC lattice. Scale bar is $100 \mathrm{~nm}$.
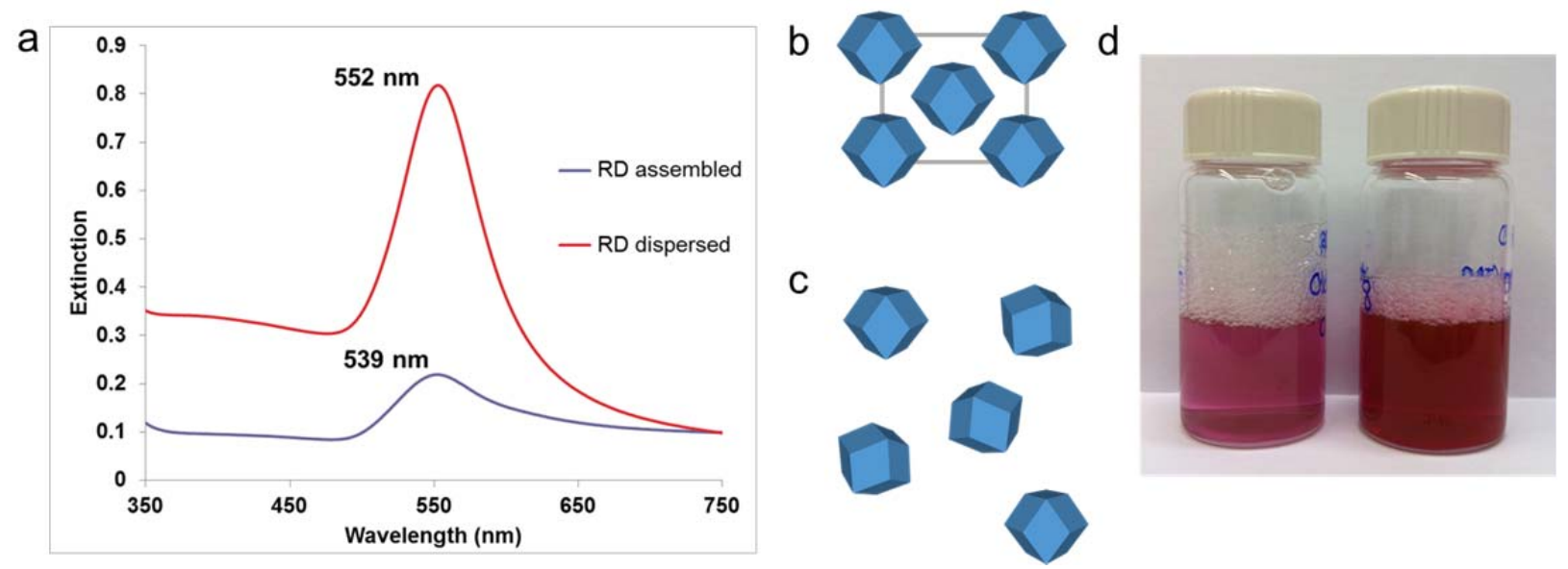

Figure S2. a) UV-Vis of the RD assembled (blue curve) and dispersed in solution by diluting the surfactant concentration in half (red curve) showing that the surface plasmon resonance (SPR) for the assembled RD dampens and blue shifts by $\sim 13 \mathrm{~nm}$. Scheme of the RD assembled into an FCC lattice (b) and dispersed (c). d) Photograph of a solution of assembled (left) and dispersed (right) RD. The reason for the blue-shifted SPR when the RD assemble in solution is currently under investigation. Assemblies of truncated cubes, octahedra, and THH that form at surfactant concentrations of $100 \mathrm{x} \mathrm{cmc}$ have not been observed to display a shifted SPR. 


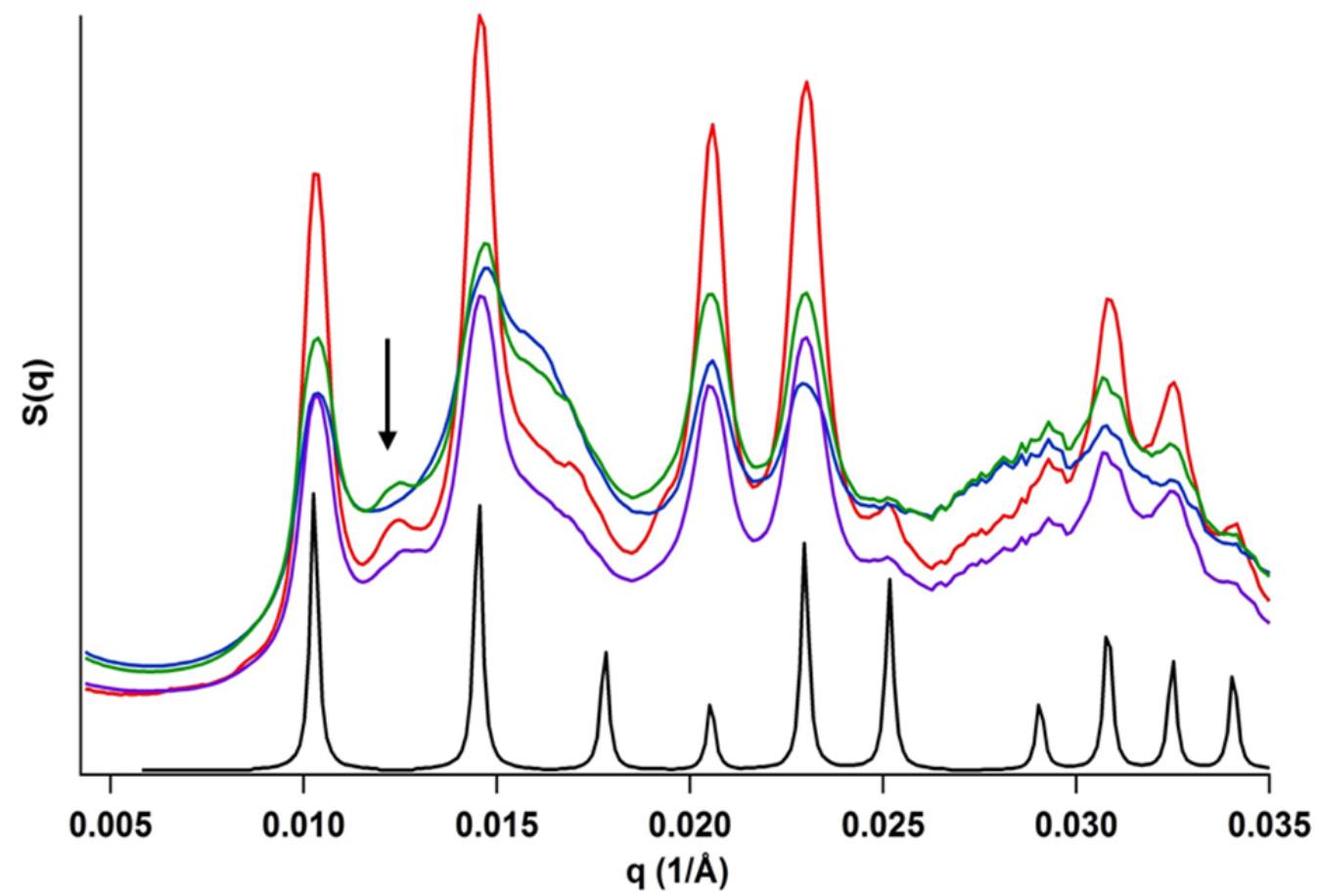

Figure S3. SAXS profiles of the truncated cube superlattices in solution. The red, green, purple, and blue traces were collected at four different locations within the capillary containing the sample. The peak in between the first and second order peaks (denoted with an arrow) does not match with the modeled SAXS pattern for a simple cubic lattice. Unlike the rest of the peaks, the location and intensity of this peak changes with location. It is believed that the peak originates from a minor domain with a slightly distorted simple cubic structure (i.e. with the (100) facets translated or rotated relative to one another).

a

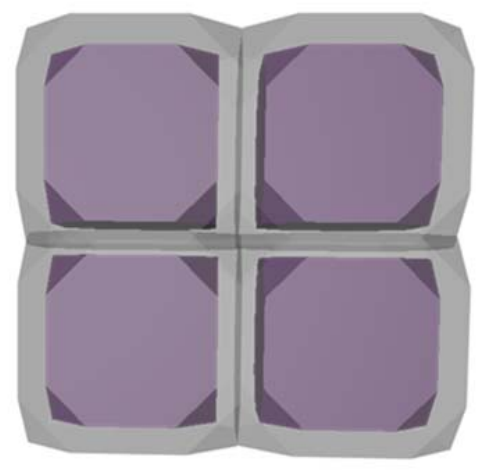

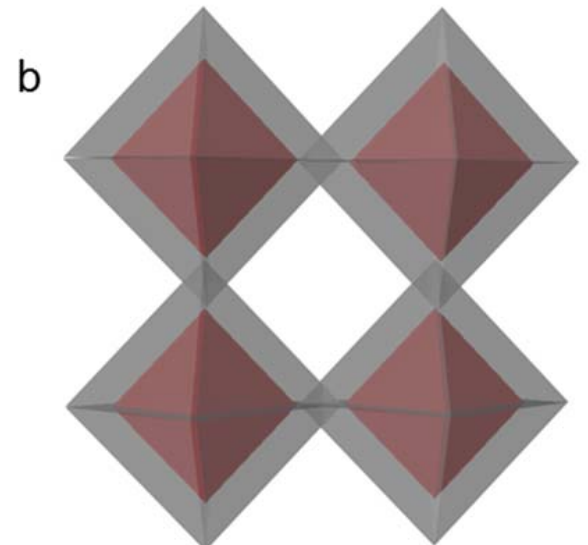

Figure S4. Schematic of the overlap of the depletion zones (denoted in grey) when truncated cubes (a) and octahedra (b) assemble in solution to illustrate the effects of electrostatic repulsion. When four truncated cubes assemble into a SC lattice, they form a pore where their truncated corners come together. The extra volume results in the particles being "pulled together" and leads to a smaller gap. When octahedra assemble into a BCC lattice, the depletion zones at their vertices overlap, leading to a greater repulsion between particles and a larger gap. 

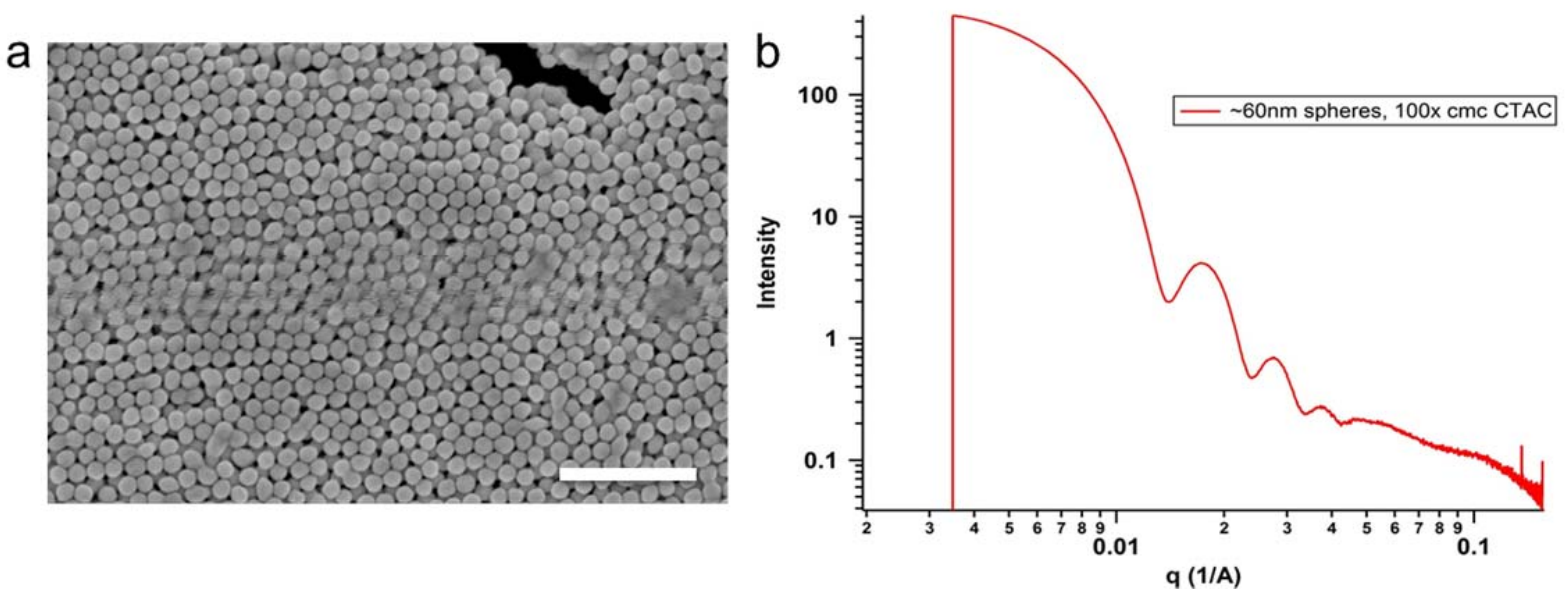

Figure S5. a) SEM and b) SAXS pattern of $60 \mathrm{~nm}$ diameter gold spheres in a solution of $0.08 \mathrm{M} \mathrm{CTAC}$ $(100 \mathrm{x} \mathrm{cmc})$. The SAXS pattern does not contain any structure factor, confirming that the spheres do not assemble into an FCC lattice in solution under the same conditions used to assemble rhombic dodecahedra, truncated cubes, octahedra, and tetrahexahedra in this work. 
a

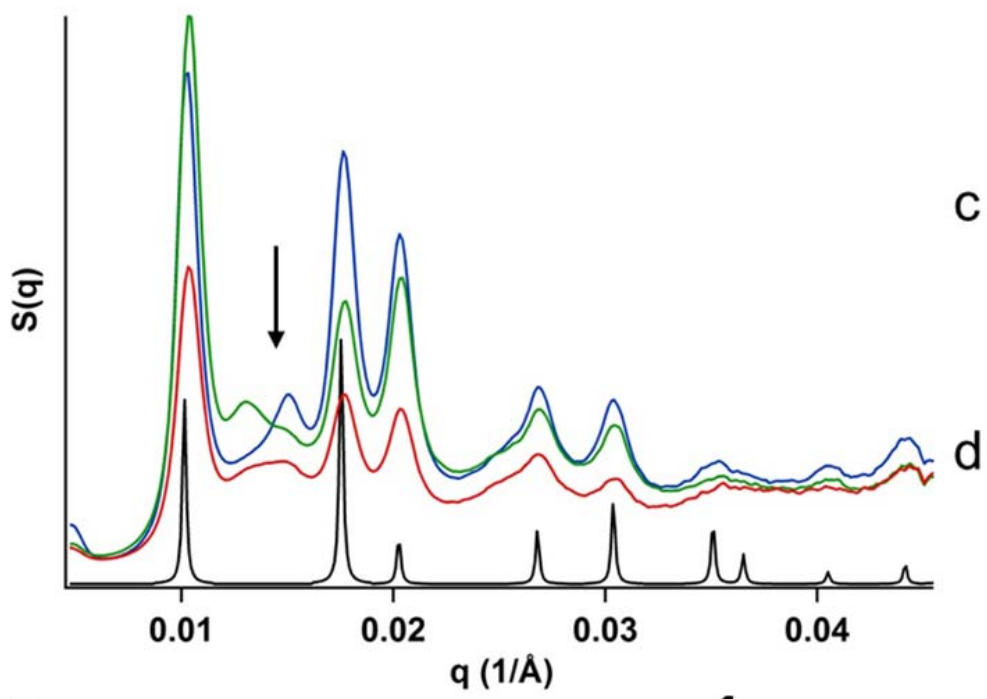

b

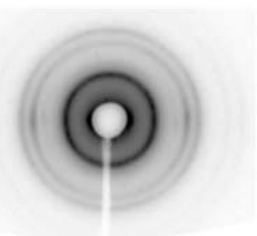

$\mathrm{C}$

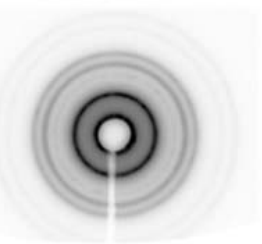

e

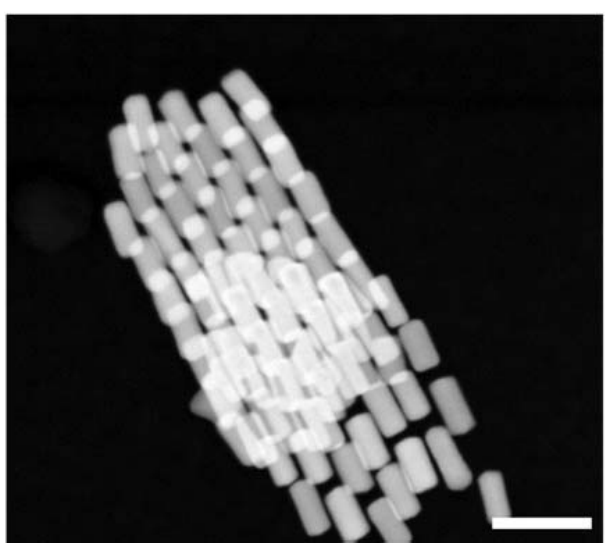

f

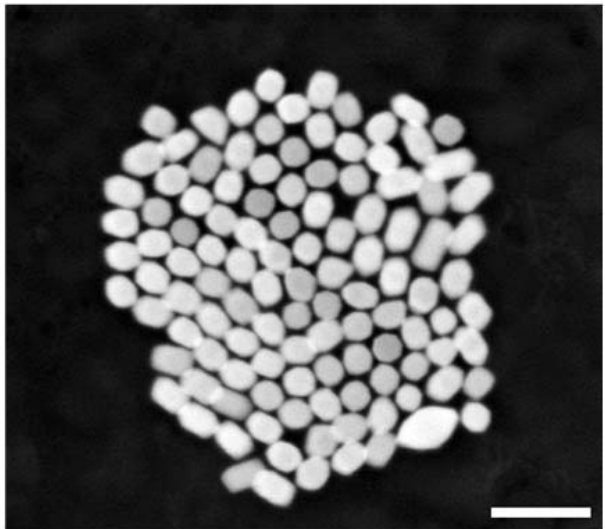

Figure S6. a) 1-D SAXS profiles of the THH superlattices in solution. The red, blue, and green traces were collected at three different locations within the capillary containing the sample (reproduced from Fig 4a in manuscript). The 2-D scattering patterns corresponding to the red (b), blue (c), and green (d) traces in a), respectively. Cryo-electron microscopy images of the THH assemblies from a side view (e) and a top-down view (f). Scale bars are $200 \mathrm{~nm}$. 


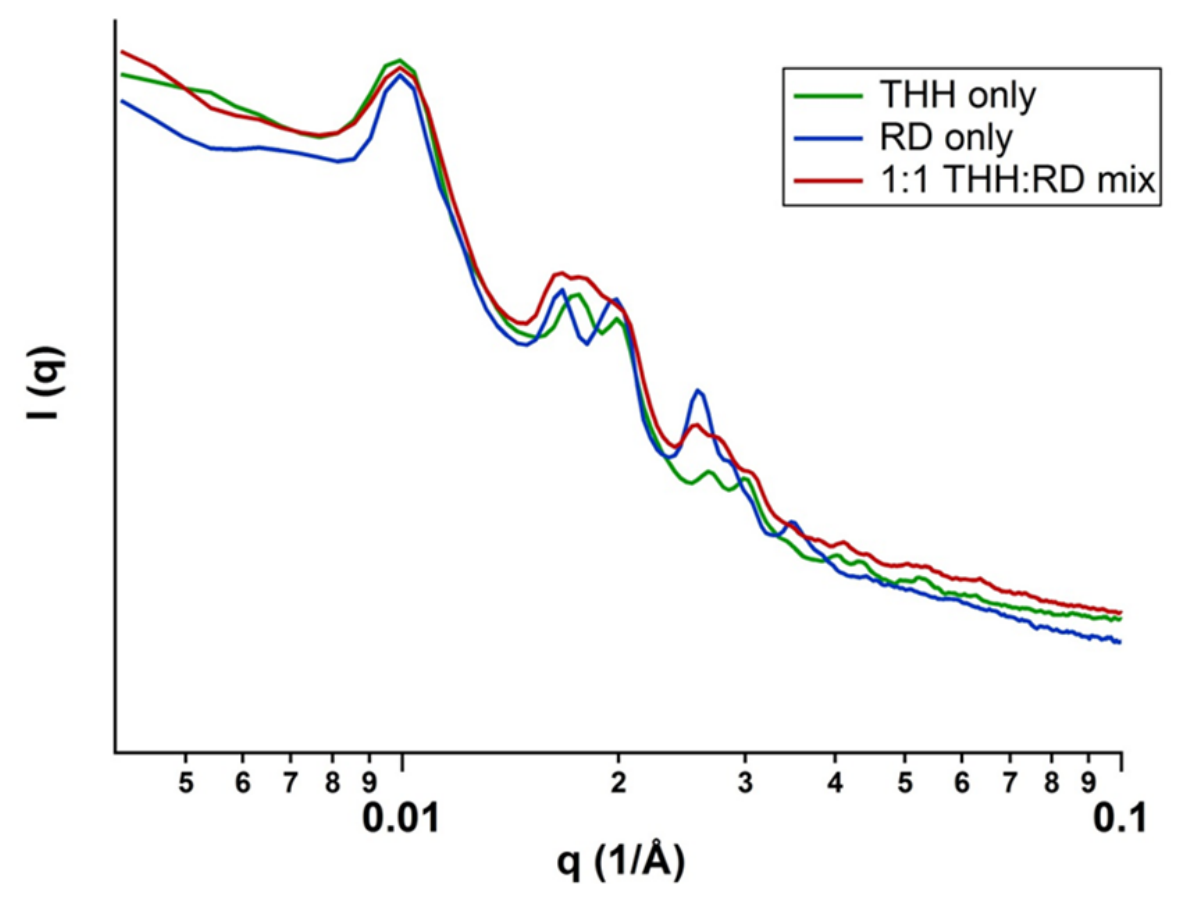

Figure S7. SAXS pattern of hexagonal THH (green trace), rhombic dodecahedra in an FCC lattice (blue trace), and a 1:1 mixture of THH and RD (red trace). The SAXS pattern of the mixture contains peaks indicating both hexagonal and FCC structures, confirming that the THH and RD phase separate when mixed in order to maximize their face-to-face alignment.
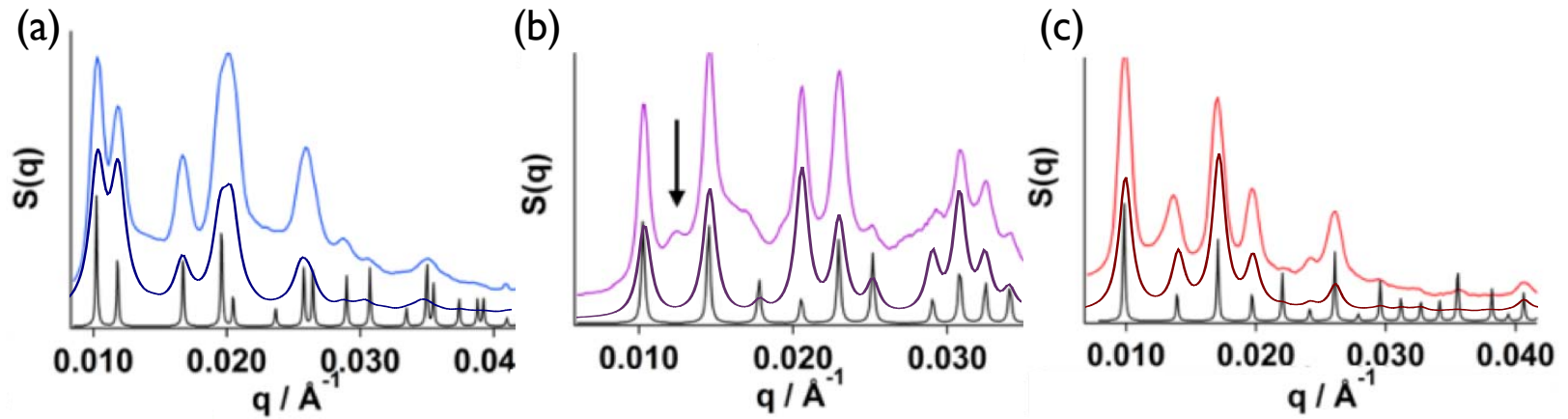

Figure S8. Modeling of powder diffraction curves using Monte Carlo integration over the atom density in the polyhedral particles (dark colored curve), using point scatterers positioned at the particle centers (solid black curves) and comparison with SAXS data (light colored curves). (a) Peak intensities match very well for rhombic dodecahedra. (b) Peak intensities only match partially well for truncated cubes. The discrepancy could be explained by the presence of vacancies and positional disorder, as frequently found in Monte Carlo simulations of hard truncated cubes. (c) Peak intensities match well for octahedra. The discrepancy could be explained by (partial) orientational disorder. 

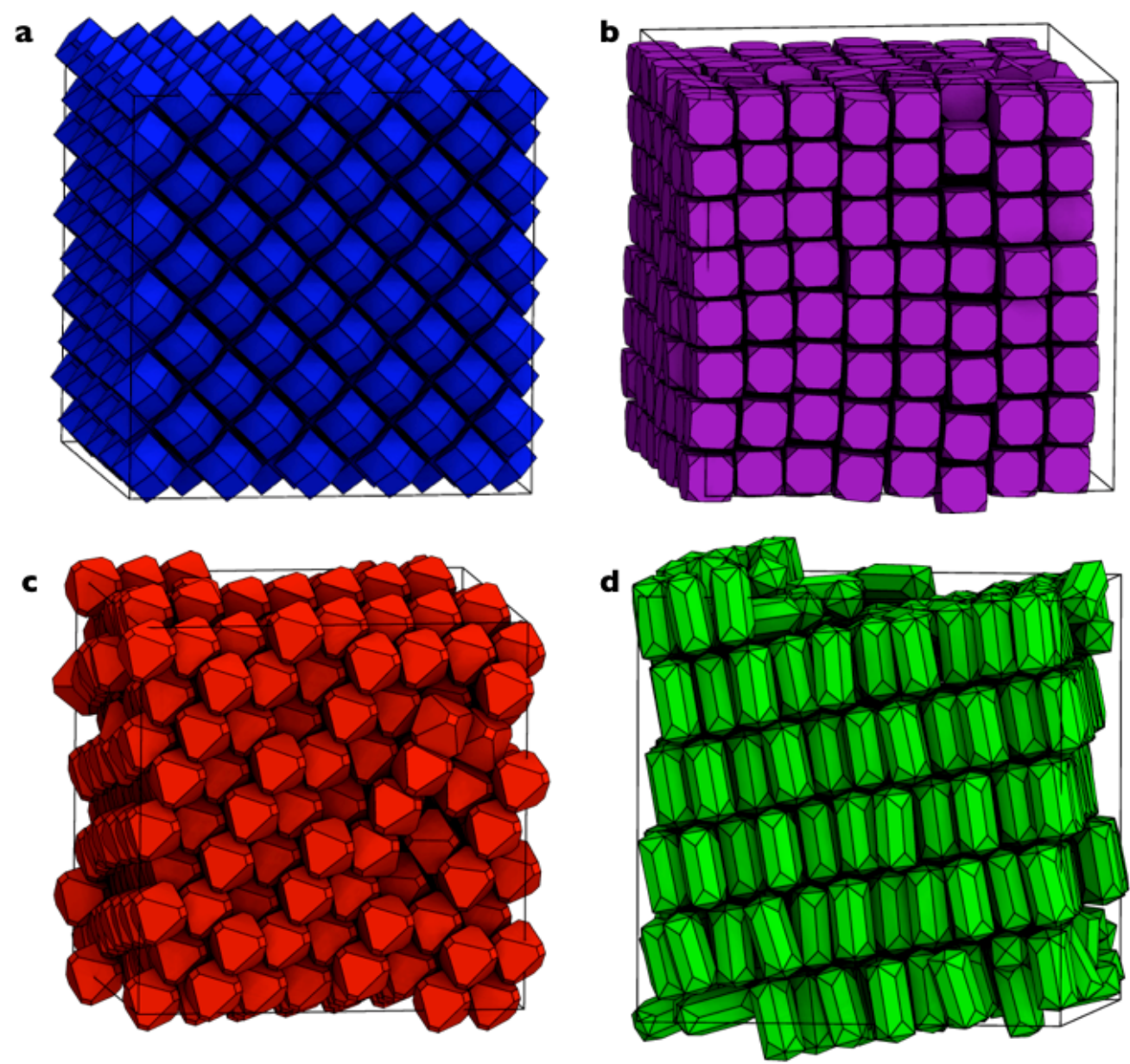

Figure S9. Monte Carlo simulation results showing the self-assembly of (a) FCC from rhombic dodecahedra, (b) simple cubic crystal from truncated cubes, (c) BCC from octahedra with slight truncation (see text), and (d) hexagonal lamellae from tetrahexahedra. In all cases entropy is maximized by these structures, which optimize face-to-face alignment and overlap of the depletion zones.

References

[1] T. Ming, W. Feng, Q. Tang, F. Wang, L. Sun, J. Wang, C. Yan, J. Am. Chem. Soc. 2009, 131, 16350-16351.

[2] M. L. Personick, M. R. Langille, J. Zhang, N. Harris, G. C. Schatz, C. A. Mirkin, J. Am. Chem. Soc. 2011, 133, 6170-6173.

[3] M. R. Langille, M. L. Personick, J. Zhang, C. A. Mirkin, J. Am. Chem. Soc. 2012, 134, 1454214554.

[4] R.-J. Roe, Methods of X-ray and Neutron Scattering in Polymer Science, Oxford University Press, New York, 2000.

[5] R. J. Macfarlane, B. Lee, M. R. Jones, N. Harris, G. C. Schatz, C. A. Mirkin, Science 2011, 334, 204-208.

[6] B. D. Cullity, S.R. Stock, Elements of X-ray Diffraction, Prentice Hall, 2001.

[7] M. R. Jones, R. J. Macfarlane, B. Lee, J. Zhang, K. L. Young, A. J. Senesi, C. A. Mirkin, Nat. Mater. 2010, 9, 913-917. 